\title{
ANGULAR CORRELATIONS OF THE X-RAY BACKGROUND AND CLUSTERING OF EXTRAGALACTIC X-RAY SOURCES
}

\author{
L. Danese ${ }^{1}$, L. Toffolatti ${ }^{2}$, A. Franceschini ${ }^{2}$, \\ J.M. Martín-Mirones ${ }^{3,2}$ and G. De Zotti ${ }^{2}$ \\ ${ }^{1}$ Dipartimento di Astronomia, Vicolo dell'Osservatorio 5, I-35122 Padova, Italy \\ ${ }^{2}$ Osservatorio Astronomico, Vicolo dell'Osservatorio 5, I-35122 Padova, Italy \\ ${ }^{3}$ Departamento de Física Moderna, Universidad de Cantabria, Avda. de Los Castros \\ s/n, E-39005 Santander, Cantabria, Spain (permanent address)
}




\begin{abstract}
The information content of the autocorrelation function (ACF) of intensity fluctuations of the $\mathrm{X}$-ray background (XRB) is analyzed. The tight upper limits set by ROSAT deep survey data on the ACF at arcmin scales imply strong constraints on clustering properties of $\mathrm{X}$-ray sources at cosmological distances and on their contribution to the soft XRB. If quasars have a clustering radius $r_{0}=12-20 \mathrm{Mpc}$ $\left(H_{0}=50\right)$, and their two point correlation function, $\xi(r)$, is constant in comoving coordinates $(\epsilon=-1.2)$, as indicated by optical data, they cannot make up more 40$50 \%$ of the soft XRB (higher contributions corresponding to lower $r_{0}$ ); the maximum contribution may reach $80 \%$ in the case of stable clustering $(\epsilon=0)$. If $r_{0} \geq 12 \mathrm{Mpc}$, a slow decrease of the $\xi(r)$ of the AGNs with increasing redshift $(\epsilon \simeq-3)$ is ruled out since it would imply an implausibly low contribution to the XRB. Active Starforming (ASF) galaxies clustered like normal galaxies $\left(r_{0} \simeq 12 \mathrm{Mpc}\right)$ can yield up to $20 \%$ or up to $40 \%$ of the soft XRB for $\epsilon=-1.2$ or $\epsilon=0$, respectively.

The ACF on degree scales, typical of existing hard X-ray surveys, essentially reflects the clustering properties of local sources and is proportional to their volume emissivity. The upper limits on scales of a few degrees imply that hard X-ray selected AGNs have $r_{0} \leq 25 \mathrm{Mpc}$ if $\epsilon=0$ or $r_{0} \leq 20 \mathrm{Mpc}$ if $\epsilon=-1.2$. No significant constraints are set on clustering of ASF galaxies, due to their low local volume emissivity. The possible signal on scales $\geq 6^{\circ}$, if real, may be due to AGNs with $r_{0} \simeq 20 \mathrm{Mpc}$; the contribution from clusters of galaxies with $r_{0} \simeq 50 \mathrm{Mpc}$ is a factor $\simeq 2$ lower. Other classes of sources clustered like normal galaxies could only account for such signal if their local volume emissivity is $\approx 6 \times 10^{38} \mathrm{erg} \mathrm{s}^{-1} \mathrm{Mpc}^{-3}$. This value is somewhat in contrast with larger estimates based on alternative methods, and implies that the bulk of the XRB is not local.
\end{abstract}


We have also computed the expected ACF in the 2-10 keV energy band on arcminute scales, that will be useful for comparison to the soon coming data from the ASTRO-D satellite.

Although the nature of sources producing the bulk of the soft and of the hard $\mathrm{XRB}$ is likely to be different, their clustering properties appear to be not much different from those of normal galaxies.

Subject headings: cosmology — galaxies: clustering — quasars - X-ray: general 


\section{INTRODUCTION}

A good deal of information on the origin of the X-ray background (XRB) as well as on the evolution of clustering in the Universe is imprinted in the angular distribution of the XRB. Studies of fluctuations and of their AutoCorrelation Function $(\mathrm{ACF})$ as well as of the cross-correlation of the XRB with source populations selected in optical, IR and radio bands are useful in constraining counts, evolution and clustering properties of extragalactic X-ray sources.

Marshall et al. (1980) pointed out that their data on the spectrum of the XRB in the energy interval 3-60 keV (henceforth HXRB) are well described by thermal bremsstrahlung emission at a temperature $T \simeq 40 \mathrm{keV}$. On the other hand the spectrum of the XRB below $3 \mathrm{keV}$ (henceforth SXRB) is still subject of debate (see e.g. McCammon \& Sanders, 1990). Nevertheless it is generally accepted that the SXRB exceeds the extrapolation of the HXRB (Wu et al. 1990; Micela et al. 1991; Hasinger 1992) and that at energies lower than $1 \mathrm{keV}$ the galactic contribution is increasingly important.

Only at soft energies ( $E \leq 3 \mathrm{keV}) \mathrm{X}$-ray imaging capabilities have already attained resolutions of few tens of arcsecond, while at harder energies collimators allow resolutions of few degrees at most. Correspondingly, at soft energies it has been possible to detect sources fainter by almost 4 orders of magnitude than those detected at hard energies. For instance, ROSAT Deep Surveys show that about $40 \%$ of the SXRB is contributed by sources brighter than $\mathrm{S}(0.5-2 \mathrm{keV}) \geq 7 \times 10^{-15} \mathrm{erg} \mathrm{s}^{-1} \mathrm{~cm}^{-2}$ (Hasinger, Schmidt \& Trümper 1991; Shanks et al. 1991; Anderson et al. 1992), while only a few percent of the HRXB has already been resolved into sources. However it is worth noticing that in the next future high resolutions will also be available at higher energies; in particular ASTRO-D is expected to produce maps with resolution of about $2^{\prime}$ in the $0.5-10 \mathrm{keV}$ energy range (Inoue 1992). On the other hand there is increasing evidence that the spectral properties of the source populations detected 
by deep surveys at soft energies are rather different from those required to account for the HXRB (see Franceschini et al. 1992, for a detailed discussion). For these reasons in the following we will discuss the autocorrelations of the SXRB and of the HXRB separately.

The XRB ACF is an integrated view of the clustering properties of the source populations contributing to the XRB. Thus studies of autocorrelations provide an important tool to explore clustering evolution since the formation of structures in the Universe (Wolfe \& Burbidge 1970; Schwartz et al. 1971; Fabian 1972). Depending on energy bands, on limiting fluxes and on angular scales, the clustering properties of different X-ray source populations can be elicited. On the other hand the clustering properties of galaxies, galaxy clusters and QSOs have been studied in optical, farIR and radio bands. Therefore it is quite informative to compare the expected contribution of these populations to the observed ACF of the XRB, looking for constraints on clustering and/or emissivity of the sources.

Studies on ACF of the XRB have been worked out by many authors, exploiting the different available data bases.

As for the SXRB, Barcons and Fabian (1989) derived the ACF on angular scales in the range $1^{\prime}$ to $15^{\prime}$, using extremely deep exposures taken with the Imaging Proportional Counter (IPC) on board of the Einstein Observatory. Their nominal energy band is $1-3 \mathrm{keV}$. On similar scales and energy band $(0.8-3.5 \mathrm{keV})$ and using again extremely deep IPC exposures Soltan (1991) has found quite stringent upper limits to the ACF on angular scales ranging from 2 to 4 arcminutes. More recently deep pointed ROSAT observations showed that the SXRB in the $0.5-2 \mathrm{keV}$ band is actually very smooth on arcminute scales. (Hasinger, Schmidt \& Trümper 1991; Hasinger 1992; Georgantopoulos et al. 1991; Carrera \& Barcons 1992). The ensuing limits on the clustering as well as on the contribution to the SXRB of the X-ray sources are quite interesting and competitive with the limits obtained from optical surveys (see Danese, De Zotti \& Andreani, 1992 for a review). 
Concerning the ACF of the HXRB, Persic et al. (1989) have derived upper limits for angular separations ranging from $3^{\circ}$ to $27^{\circ}$ by analyzing HEAO 1 A-2 data in the nominal 2-10 keV band; the ensuing constraints have been discussed by De Zotti et al. (1990). Carrera et al. (1991) explored the ACF on similar energy interval (4-12 keV) and angular scales using exposures obtained with the Large Area Counter (LAC) on board of the Ginga satellite and found results consistent with those of Persic et al. (1989). Martín-Mirones et al. (1991) and Carrera et al. (1992) studied the ACF on sub-degree angular scales using A-2 and LAC data respectively. Again the results of both analyses are fully consistent and have been used to put significant constraints on clustering of AGNs and galaxy clusters.

Extending the analysis to a larger portion of the sky covered by the A-2 experiment, Mushotzky \& Jahoda (1992) have reported a possible detection of positive autocorrelation at scales in the range $6^{\circ}$ to $20^{\circ}$.

In this paper we will exploit the whole body of data to examine the limits on clustering, clustering evolution and volume emissivity of X-ray sources contributing to the SXRB and HXRB. In Section 2 the theory of the ACF is presented and the effects of the characteristics of individual experiments on the results are briefly discussed. In Section 3 and 4 we present the constraints on emissivity and clustering properties of AGNs and galaxies following from the current limits on ACF of the SXRB and HXRB respectively. Section 5 is devoted to the discussion and conclusions.

A Hubble constant $H_{0}=50$ and a cosmological deceleration parameter $q_{0}=0.5$ are used throughout the paper.

\section{THE ANGULAR AUTOCORRELATION FUNCTION}

Cell-to-cell fluctuations of randomly distributed or clustered unresolved sources produce intensity fluctuations $\delta I(\mathbf{n})=I(\mathbf{n})-\langle I\rangle$ of the observed background. The 
angular ACF of the intensity fluctuations is usually defined as

$$
W(\theta)=\frac{\left\langle\delta I(\mathbf{n}) \delta I\left(\mathbf{n}^{\prime}\right)\right\rangle}{\langle I\rangle^{2}},
$$

where $\theta$ is the angle between the directions $\mathbf{n}$ and $\mathbf{n}^{\prime}$, and the spatial average $\langle I\rangle$ is referred to the residual background, once the detected sources have been subtracted. The contribution to the ACF from clustered source populations can be computed under rather general hypotheses (see e.g. Martín-Mirones et al. 1991). If the maximum clustering scale $r_{\max }$ is much smaller than the Hubble radius, $c / H_{\circ}$, and the maximum value of angular separation is much less than one radian, then the proper separation $r$ between points on two lines of sight separated by an angle $\theta$ can be approximated by:

$$
r=\left[(c \delta t)^{2}+\left(d_{A} \theta\right)^{2}\right]^{1 / 2},
$$

where $d_{A}=d_{L}(1+z)^{-2}$ is the angular diameter distance, and $\delta t=(d t / d z) \delta z=$ $-H_{o}^{-1}(1+z)^{-2}(1+\Omega z)^{-1 / 2} \delta z$. The luminosity distance is given by $d_{L}=$ $\left(2 c / \Omega^{2} H_{0}\right)\left\{\Omega z+(\Omega-2)\left[-1+(\Omega z+1)^{1 / 2}\right]\right\}$.

With the additional hypothesis that the beams [response function $f(\vartheta, \varphi)$ ] do not overlap, Martín-Mirones et al. (1991) have shown that the ACF as a function of the angular separation $\theta$ is given by:

$$
\begin{array}{r}
W(\theta)=\left(\frac{c}{4 \pi H_{o}\langle I\rangle}\right)^{2} \int d \omega f(\vartheta, \varphi) \int d \bar{\omega} f(\bar{\vartheta}, \bar{\varphi}) . \\
\cdot \int_{z_{m}\left(L_{\min }, S_{l}\right)}^{z_{\max }} d z \frac{j_{\text {eff }}^{2}(z)}{(1+z)^{4}(1+\Omega z)} \int_{\max \left[z_{m}-z,-\Delta\left(r_{\max }\right)\right]}^{\min \left[z_{\max }-z, \Delta\left(r_{\max }\right)\right]} d(\delta z) \xi(r, z),
\end{array}
$$

where $\Delta\left(r_{\max }\right)$ is the value of $\delta z$ corresponding to the maximum scale of clustering, and

$$
j_{e f f}(z)=\int_{L_{\min }}^{\min \left[L_{\max }, L\left(S_{l}, z\right)\right]} d \log L L n_{c}(L, z) K(L, z)
$$

is the effective volume emissivity; $L\left(S_{l}, z\right)$ is the luminosity of a source at the redshift $z$ that yields a flux equal to the detection limit $S_{l}$, whereas $z_{m}\left(L_{m i n}, S_{l}\right)$ is the redshift 
at which a source of minimum luminosity $L_{\min }$ has the limiting flux $S_{l}$ and $z_{\max }$ is the upper limit to the redshift of sources. The average $\langle I\rangle$ is performed after subtracting sources brighter than $S_{l}$.

The epoch-dependent Luminosity Function of the clustered sources is $n_{c}(L, z) d \log L, \xi(r, z)$ is the epoch-dependent spatial correlation function and the $\mathrm{K}$-correction factor is given by

$$
K(L, z)=\frac{\int_{E_{1}}^{E_{2}} L[E(1+z), z] d E}{\int_{E_{1}}^{E_{2}} L(E, 0) d E},
$$

$E_{1}$ and $E_{2}$ bounding the relevant energy band.

Available data suggest that $\xi(r, z)$ can be represented as (see Peebles 1980; Bahcall \& Soneira 1983; Sebok 1986)

$$
\begin{gathered}
\xi(r, z)=D^{2}(z) \xi_{0}(r) \\
\xi_{0}=\left(r_{0} / r\right)^{\gamma}
\end{gathered}
$$

with $\gamma \simeq 1.8$ for a number of populations of extragalactic sources.

Actually, in numerical calculations a more refined model is used, that make the correlation function flat at small radii and explicitly incorporate a maximum radius $r_{\max }$, such as $\xi=0$ if $r>r_{\max }$. In the following we will adopt $r_{\max }=3 r_{0}$.

The factor $D^{2}(z)$ allows for clustering evolution and has been often parametrized as

$$
D^{2}(z)=(1+z)^{-(3+\epsilon)}
$$

The case $\epsilon=-3$ corresponds to the very peculiar situation of $r_{0}$ constant in physical coordinates, while $\epsilon=\gamma-3$ implies $r_{0}$ constant in comoving coordinates. The case of linear growth of the clustering is represented by $\epsilon=\gamma-1$ if $\Omega=1$ (Martínez-Gonzales \& Sanz 1991). A self-similar evolution of the correlation function (requiring $\Omega=1$ and a power-law spectrum of initial density perturbations) yields 
$\epsilon=0$. In the latter case, on scales where $\xi \gg 1$ the number of object in a physical volume is constant (statistically stable clustering). Galaxy formation models like the "pancake" model and biased CDM models predict enhanced clustering of high redshift and luminous QSOs (Rees 1986; Kaiser 1986; Efstathiou \& Rees 1988).

The redshift dependence of the ACF is determined by the angular scale, the clustering evolution and the emissivity of the unresolved sources. Figure 1 and 2 emphasize the different redshift dependence of the emissivity, $j_{e f f}$, and of the ACF, $W(\theta)$, for some relevant angular separations, clustering scales and evolution. The emissivities have been computed using local luminosity functions and cosmological evolutions consistent with the available soft and hard X-ray data (see below and Franceschini et al. 1992). It is apparent that different combinations of clustering scales and angular separations weight differently the volume emissivity. For instance, large angular scales, typical of hard $\mathrm{X}$-ray collimators $\left(\theta>2^{\circ}\right)$, tend to elicit the clustering properties of local sources. Panels 1b) and 1c) show that for angular separations of $\gtrsim 2^{\circ}$ the effect of clustering evolution is important, even for cases in which the physical clustering scale is significantly decreasing with increasing redshift, e.g. $\epsilon \geq-1.2$. On the other hand the effect is negligible in the cases of separations larger than $4^{\circ}$ and with $r_{0} \leq 20 \mathrm{Mpc}$, because only nearby objects contribute to the ACF (panel 1a).

In the soft bands, X-ray telescopes can explore small scales (few arcminutes) and detect sources down to faint limiting fluxes; for instance, in deep ROSAT fields up to $50 \%$ of the SXRB has already been resolved (Hasinger 1992). Small angular separations make the ACF more sensitive to the clustering of high redshift sources, as can be seen by comparing Figure 1 with Figure 2. In particular, the cases reported in panels $c$ ) and $d$ ) of Figure 2 have been computed with redshift dependences of the volume emissivity very similar to those used in Figure 1. Figure 2 shows that on arcminute scales, the current resolution achieved by soft bands telescopes, the redshift dependences of the ACF and of the volume emissivity are very similar. The different 
relationship between the ACF passing from degree to arcminute scales, implies that while the observed HXRB ACF feels only the volume emissivity of local sources, that of the SXRB comes from the integrated contribution to the XRB of sources at intermediate and high redshift.

Thus, evolution models for the volume emissivity of clustered sources yielding the same fraction of the background but with a different redshift dependence could produce rather different values of the ACF at some angular scales.

The dependence of the ACF on the angular scale $\theta$, on the clustering scale $r_{0}$ and on the fraction $f$ of the residual background produced by the unresolved sources is rather simple in the case that the beam angular separation $\theta$ and the FWHM of the beam $\theta_{\text {beam }}$ are much smaller than the minimum angle $\theta\left(r_{0}, z\right)$ subtended by the physical clustering scale. Then the innermost integration in equation (3) can be extended from $-\infty$ to $\infty$ and we get (De Zotti et al. 1990, eq. 20):

$$
W(\theta) \propto \theta^{1-\gamma} r_{0} f^{2}
$$

This relationship holds in the case of ACF studies on small angular scales; presently this only is the case of soft $\mathrm{X}$-ray surveys, however in the next future ACF on small angular scales should also be available at harder energies from observations performed with ASTRO-D.

So far the ACF derived from available observations in hard X-ray bands, referring to large angular separations $\left(\theta \geq 2^{\circ}\right)$, mostly reflects (particularly in the case of a steep clustering evolution: $\epsilon \approx 0)$ the clustering properties of rather local $(z \ll 1)$ sources which give a small contribution to the background. In this case, the scaling law is similar to equation (9), but with $f$ replaced by the ratio of the local volume emissivity of sources to that of the XRB, $j_{\text {sources }} / j_{\mathrm{XRB}}$. 


\section{FROM THE SXRB ACF}

Hasinger, Schmidt \& Trümper (1991) and Hasinger (1992 and private communication) have derived from deep ROSAT survey data tight $3 \sigma$ upper limits to the ACF on arcmin scales: $W\left(2^{\prime}-3^{\prime}\right) \leq 8 \times 10^{-3}$ and $W\left(9^{\prime}-10^{\prime}\right) \leq 2 \times 10^{-3}$, in the 0.9-2.4 keV band, after subtracting sources brighter than $S_{l} \approx 5 \times 10^{-15} \mathrm{erg} \mathrm{cm}^{-2} \mathrm{~s}^{-1}$. Comparable upper limits on the same scales have been found by preliminary analyses of ROSAT surveys on different areas by Carrera \& Barcons (1992) and by Georgantopoulos et al. (1992). Analyzing deep Einstein Observatory IPC fields in the energy band 0.8-3.5 keV, Soltan (1991) derived an even tighter upper limit, $W\left(2^{\prime}-5^{\prime}\right) \leq 3 \times 10^{-3}$, excluding sources brighter than $S_{l} \approx 5 \times 10^{-14} \mathrm{erg} \mathrm{cm}^{-2} \mathrm{~s}^{-1}$; this limit, however, may be too optimistic (see Barcons \& Fabian 1989 and Carrera \& Barcons 1992).

As shown by the ROSAT deep survey in the QSF3 field (Shanks et al. 1991) the large majority of the $\mathrm{X}$-ray sources brighter than $S_{l}(0.5-2 \mathrm{keV}) \approx 1 \times$ $10^{-14} \mathrm{erg}^{-2} \mathrm{~cm}^{-1}$ are quasars at substantial redshifts. As a consequence AGNs are expected to give an important contribution to the source counts even below this limiting flux, although other source populations like Active Star Forming (ASF) galaxies could start to appear. Since it is reasonable to assume that AGNs and ASF galaxies may significantly contribute to the residual SXRB, the following discussion will be focused on their clustering and emissivity properties. Although galaxy clusters are practically absent in the deep surveys, there are models predicting that clusters could give from 10 to $20 \%$ of the SXRB (Blanchard et al. 1992; Cen \& Ostriker 1992; Cavaliere, Menci \& Burg 1992). Note that, since these are expected to be extended, low redshift and low brightness objects, their contribution to the autocorrelations on few arcminute scales could not be negligible, as in the case of the NEP 'blotch' (Hasinger, Schmidt \& Trümper 1991). 


\section{1. $A G N s$}

In Figure 3 we have reported the constraints on the fraction of the total background contributed by unresolved AGNs and on the corresponding clustering scale, $r_{0}$, obtained adopting the ROSAT $3 \sigma$ upper limits on the ACF for $9^{\prime}-10^{\prime}$ scales. Similar - albeit somewhat less stringent - results can be found comparing model predictions with limits on $2^{\prime}-4^{\prime}$ scales.

To avoid a too large contamination by the galactic background, the analysis of the autocorrelations has been limited to data in the energy range $0.9 \leq E \leq$ $2.4 \mathrm{keV}$, though ROSAT is sensitive down to softer energies (Hasinger 1992). Henceforth by SXRB we mean the $\mathrm{X}$-ray background in the energy range $1 \lesssim E \lesssim 3$ $\mathrm{keV}$. The background intensity in the 0.9-2.4 keV band has been assumed to be $2 \times 10^{-8} \mathrm{erg} \mathrm{cm}^{-2} \mathrm{~s}^{-1} \mathrm{sr}^{-1}$, following Hasinger (1992) and consistent with McCammon $\&$ Sanders (1990). From table 1 of Hasinger (1992) we have also deduced that sources

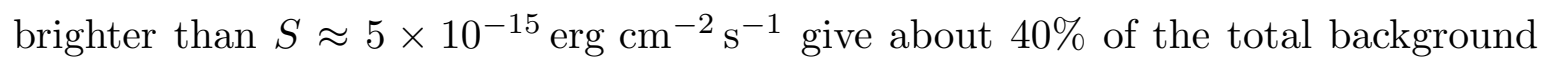
and about $25 \%$ of it is attributable to AGNs (Shanks et al. 1991).

The calculations have been done assuming for the sources a power law spectrum $\left(F \propto E^{-\alpha}\right)$ with spectral index $\alpha=1.2$ as suggested by direct measurements of bright soft $\mathrm{X}$-ray selected sources and by the average source spectra observed by ROSAT. We have also used the Local Luminosity Function derived by Maccaccaro et al. (1991) and evolution models that fit the EMSS (Gioia et al. 1990) and ROSAT Deep surveys counts (Hasinger et al. 1991, Shanks et al. 1991, Anderson et al. 1992, Franceschini et al. 1992). With these assumptions the fraction of the SXRB contributed by unresolved AGNs is somewhat dependent on the assumed spectra, luminosity functions and evolution. Therefore we have decided to keep this fraction as a free parameter, subject only to the condition that the corresponding redshift dependence of the emissivity of undetected AGNs is a smooth extrapolation of that fitting the available data. The conclusions also depend on the geometry of the 
Universe in the sense that changing from $q_{0}=0.5$ to $q_{0}=0$ the allowed total fraction of the contributed background increases by about $\sim 30-40 \%$.

It is easy to see from Figure 3 that weak clustering evolution $(\epsilon \leq-1.2)$ would produce rather large autocorrelations even with a small clustering scale $r_{0}$. As a consequence, if AGNs cluster like galaxies, $r_{0} \simeq 12 \mathrm{Mpc}$, the total AGN contribution to the SXRB is bound to be less than $\sim 50 \%(\sim 25 \%$ from detected plus $\sim 25 \%$ from unresolved sources) in the case $\epsilon=-1.2$ and less than $\sim 35 \%$ in the case $\epsilon=-3$. Because any reasonable model that fits ROSAT deep counts and EMSS counts predicts a total AGN contribution at least as large as $30-35 \%$ and since a minimum value $r_{0}=12 \mathrm{Mpc}$ has been found for optically selected QSOs (Boyle 1991; Andreani \& Cristiani 1992), clustering evolution must be faster than that implied by $\epsilon=-3$ (clustering constant in physical coordinates).

ROSAT results also provide a significant test of the suggestion by Bahcall \& Chokshi (1991) that the QSO correlation function is intermediate $\left(r_{0}=24 \mathrm{Mpc}\right)$ between those of galaxies and of rich clusters, as a consequence of the preferential location of QSOs in small groups of galaxies, and has an evolution parameter $\epsilon=$ -1.8. From Figure 3 it is apparent that QSOs clustered in this way must produce less than $40 \%$ of the total SXRB (QSOs below the detection limit yielding less than $15 \%)$.

On the other hand the allowed AGN contribution increases up to $80 \%$ in the case of stable clustering $(\epsilon=0)$.

It is worth noticing that AGNs with clustering scale $r_{0}=20 \mathrm{Mpc}$ and $\epsilon=-1.2$, values still allowed by optical data, are quite close to infringe ROSAT upper limits on the ACF, if they produce $40 \%$ of the SXRB.

For sake of completeness we reported in Figure 4 the constraints derived by using Soltan's (1991) limits on the ACF, although they could be too optimistic (Carrera \& Barcons 1992). At the limiting flux $S_{l} \approx 5 \times 10^{-14} \mathrm{erg} \mathrm{cm}^{-2} \mathrm{~s}^{-1}(0.8-3.5 \mathrm{keV})$ of the surveyed fields AGNs produce only $\sim 20 \%$ of the background (Primini et al. 1991). 


\subsection{Active Star Forming galaxies}

Several authors (Danese et al. 1987; Hacking, Condon \& Houck 1987; Franceschini et al. 1988) have pointed out that Active Star Forming (ASF) galaxies (galaxies with enhanced star formation rate in comparison with normal spiral galaxies) exhibit significant cosmological evolution. Intense star forming activity should show up also through X-ray emission powered by supernovae and X-ray binaries (Bookbinder et al. 1980; Stewart et al. 1982; Griffiths \& Padovani 1990). Current estimates of the contribution of the ASF galaxies to the XRB are still rather uncertain ranging from 5-10\% to 30\% (but an even larger contribution cannot be excluded), mainly because their X-ray emission is poorly known. On the other hand ROSAT deep surveys have shown that QSOs are by far the dominant population down to the faintest observed fluxes and only a few of the detected sources could possibly be identified with ASF galaxies.

We have computed the contribution to the ACF of ASF galaxies using a luminosity function and luminosity evolution $\left(L(z)=L(z=0) \times(1+z)^{2.9}\right)$ appropriate to keep the population basically undetected at the ROSAT deep survey limit and to produce an important fraction of the background. A spectral index $\alpha=1.5$ has been adopted (see Boller et al. 1992). The cumulative fractional emissivity per unit volume as a function of redshift is shown in panels $c$ ) and $d$ ) of Figure 2. The constraints imposed by the smoothness of the ACF on the ASF clustering are presented in Figure 5. If we assume a clustering scale $r_{0} \approx 12 \mathrm{Mpc}$ for these galaxies, the limits from ROSAT and from Einstein Observatory deep surveys suggest that clustering evolution keeping $r_{0}$ constant in physical coordinates would entail that ASF galaxies contribute less than $20 \%$ to the SXRB; the contribution could reach $40 \%$ in the case of substantial clustering evolution $(\epsilon=0)$. As already suggested by Danese, De Zotti \& Andreani (1992) the constraints on ASF galaxies could be alleviated only if one extends to the ASF galaxies the weak clustering found 
by Efstathiou et al. (1991) for the faint blue galaxies detected in deep CCD images (Tyson 1988).

\section{CONSTRAINTS ON X-RAY SOURCE CLUSTERING AND EMISSIVITY FROM THE HXRB ACF}

The autocorrelation function of the HXRB on scales larger than $3^{\circ}$ has been derived exploiting data obtained by the HEAO 1 A-2 experiment in the 2-10 keV band by Persic et al. (1989). More recently, using the same data base Martín-Mirones et al. (1991) extended the analysis to scales smaller than $3^{\circ}$. Similar analyses have been done by Carrera et al. (1991, 1992) who have used large sky areas scanned by the Ginga Large Area Counter in the $4-12 \mathrm{keV}$ band. It is encouraging that the analyses of both groups produce similar $3 \sigma$ upper limits on angular separations larger than $2^{\circ}$, for instance $W\left(2^{\circ}\right) \leq 7 \times 10^{-4}$.

Mushotzky \& Jahoda (1992), analysing data from the HEAO 1 A-2 experiment on an area much larger than that exploited by Persic et al. (1989), have claimed the first detection (99\% confidence level) of autocorrelations in the HXRB with $W(\theta) \simeq$ $3 \times 10^{-5}$ on angular scales ranging from 6 to 20 degrees. The upper limits found by other authors are consistent with this possible detection. All the above mentioned analyses have been done with limiting flux $S_{l} \approx 2.5 \times 10^{-11} \mathrm{erg} \mathrm{s}^{-1} \mathrm{~cm}^{-2}(2-10 \mathrm{keV})$ for source detection.

The distinction we have made between hard and soft XRB, although arbitrary to some extent, is motivated by some evidence that the SXRB is mainly contributed by sources like optically selected QSOs with steep and unabsorbed spectra (henceforth soft spectrum AGNs) and possibly by ASF galaxies, whereas the HXRB is mainly contributed by relatively low luminosity AGNs with heavily absorbed spectra (henceforth hard spectrum AGNs). The objects dominating the SXRB may give 
a minor fraction $(f \leq 20 \%)$ of the $2-10 \mathrm{keV}$ background, whereas the low luminosity, highly absorbed objects with hard spectra give a negligible contribution to the SXRB (see, for a comprehensive discussion, Franceschini et al. 1992).

As it is well known, the limits on clustering of optically selected QSOs refer to high luminosity, high redshift objects (see Boyle 1991; Andreani \& Cristiani 1992). Such limits are thus more likely to apply to AGNs contributing to the SXRB, while the clustering properties of low luminosity, heavily absorbed AGNs could be rather different.

In the following we will use data and limits on the HXRB ACF to explore clustering and X-ray emission properties of the hard spectrum AGNs, ASF galaxies and normal galaxies.

\subsection{Active Galactic Nuclei}

The constraints ensuing from the limits on the HXRB ACF at angular scales smaller than few degrees have been discussed by Martín-Mirones et al. (1991), and Carrera et al. $(1991,1992)$. The main conclusion is that the smoothness of the HXRB implies a rather small clustering scale for the AGNs, if they are required to produce a significant fraction of the background. We confirm this conclusion. In particular, using the Piccinotti et al.(1982) local Luminosity Function and luminosity evolution $L(z)=L(z=0) \times(1+z)^{2.6}$ a fraction $\sim 60 \%$ of the total background is produced; the corresponding redshift dependence of the volume emissivity is presented in Figure 1. In such a case we have found that the limits on angular scales $\theta \leq 3^{\circ}$ imply $r_{0} \leq 25$ $\mathrm{Mpc}$ in the case of stable clustering (i.e. $\epsilon=0$ ) and, $r_{0} \leq 20 \mathrm{Mpc}$ for a constant comoving clustering scale.

On the other hand the possible detection of autocorrelations on a $6^{\circ}$ scale by Mushotzky \& Jahoda (1992) would imply $r_{0} \simeq 20 \mathrm{Mpc}$, independently of the clustering evolution and of the geometry of the Universe, because the contribution 
to the $6^{\circ} \mathrm{ACF}$ of objects clustered on linear scales $r_{0} \leq 20 \mathrm{Mpc}$ is confined to low redshifts, as can be seen in Figure 1a. Actually $\sim 80 \%$ of the total autocorrelation is produced by AGNs within $z \lesssim 0.07$ giving only $\sim 1 \%$ of the total background, based on the local luminosity function by Piccinotti et al. (1982).

Clustering evolution keeping constant the physical scale $(\epsilon=-3)$ does not exclude the possibility of a relevant contribution (about 60\%) of hard spectrum AGNs to the HXRB, provided that $r_{0} \leq 12 \mathrm{Mpc}$.

The limits imposed by the HXRB autocorrelations are looser than those derived using the soft band data. Apart from the fact that ROSAT ACF studies (Hasinger 1992) refer to a residual $60 \%$ of the background, the tightness of the limits imposed by the SXRB ACF is due to the imaging capability of the telescopes that allows to explore the ACF on small angular scales. Actually, as discused in Section 2, only a small fraction of the total emissivity of the population contributes to the autocorrelations in the hard band case, because large angular scales emphasize low redshift contributions when the explored linear clustering scales are of the order of few tens of megaparsecs. Contrariwise, in the soft band the fact that the ACF can be studied on small angular scales (from few to several arcminutes) ensures that $\theta \ll \theta\left(r_{0}, z\right)$ at any redshifts and, as a consequence, that the overall source population contributes to the autocorrelations. On the other hand this fact makes the soft and hard data on the ACF complementary, because they are sensitive mainly to high and low redshift source clustering respectively.

\subsection{Active Star Forming and normal galaxies}

If we assume that the typical clustering scale of ASF galaxies is similar to that of normal galaxies $\left(r_{0} \approx 10-12 \mathrm{Mpc}\right)$, the limits on the HXRB ACF do not set any significant constraint on their contribution to the XRB, even in the case of a constant physical clustering scale, due to their low local volume emissivity. For 
example, we have assumed that ASF galaxies have a present number density $n_{\mathrm{ASF}} \approx$ $1.5 \times 10^{-3} \mathrm{Mpc}^{-3}$, close to that of bright galaxies, and an average $2-10 \mathrm{keV}$ luminosity $\left\langle L_{x}\right\rangle \approx 1 \times 10^{41} \mathrm{erg} \mathrm{s}^{-1}$. The local volume emissivity is then $\rho_{x}(2-10 \mathrm{keV}) \approx 1.5 \times$ $10^{38} \mathrm{erg} \mathrm{s}^{-1} \mathrm{Mpc}^{-3}$, a factor of 5 higher than the limit found by Rephaeli et al. (1991); with a luminosity evolution $L(z)=L(z=0) \times(1+z)^{3.4}$ about $60 \%$ of the HXRB is produced.

Even under these extreme assumptions, this source population yields autocorrelations $W\left(2^{\circ}\right) \leq 2 \times 10^{-5}$, much smaller than the observational upper limits even in the case $\epsilon=-3$. Note however that the case for a dominant contribution to the HXRB from the ASF galaxies is unlikely, because their observed spectra (see e.g. Boller et al. 1992) are much steeper than those, flat or even inverted, required to account for the HXRB after removal of contributions of the already detected sources (see Franceschini et al. 1992).

As shown in $\S 2$, the contribution to the $\mathrm{ACF}$ on a $6^{\circ}$ scale depends on the local volume emissivity. In particular with a clustering scale $r_{0}=12 \mathrm{Mpc}$, typical of galaxies, it can be seen that

$$
W\left(6^{\circ}\right) \approx 1-0.8 \times 10^{-6}\left(\frac{\rho_{x}}{10^{38}}\right)^{2},
$$

depending on the redshift the bulk of the HXRB is assumed to come from.

We conclude that ASF galaxies cannot significantly contribute to the autocorrelations reported by Mushotzky \& Jahoda (1992), even in the case they eventually produce a dominant fraction of the HXRB.

Cross-correlations of the XRB surface brightness with the projected distribution of galaxies, both optically and far-IR selected, have been used to evaluate or constrain the X-ray volume emissivity associated with galaxies (Turner \& Geller 1980; Jahoda et al. 1991; Lahav 1992; Boldt 1992; Jahoda et al. 1992). In particular Jahoda et al. (1992) using HEAO 1 A-2 data and the UGC and ESO catalogs have been able to estimate the $2-10 \mathrm{keV} \mathrm{X}$-ray luminosity density of the local Universe $\rho_{x}(2-$ 
$10 \mathrm{keV})=(12.5 \pm 7) \times 10^{38} \mathrm{erg} \mathrm{s}^{-1} \mathrm{Mpc}^{-3}$. Relating the peculiar velocity of the local group of galaxies to the total dipole moment of the all-sky distribution of the $\mathrm{X}$-ray flux, the same authors have estimated the present epoch volume emissivity $\rho_{x}(2-10 \mathrm{keV}) \sim 30 \times 10^{38}\left(b \Omega^{-0.6}\right)^{-1} \mathrm{erg} \mathrm{s}^{-1} \mathrm{Mpc}^{-3}$ (b is the bias parameter for low luminosity X-ray sources). Boldt (1992) has also estimated the 2-10 keV local volume emissivity of normal galaxies $\rho_{x}(2-10 \mathrm{keV}) \approx 3 \times 10^{37} \mathrm{erg} \mathrm{s}^{-1} \mathrm{Mpc}^{-3}$, extrapolating the results found by Fabbiano, Kim \& Trinchieri (1992) in the soft bands. The insertion of the latter value of the volume emissivity in equation (10) shows that the expected contribution from normal galaxies to the ACF at $6^{\circ}$ is negligible.

On the other hand, after equation (10), the limits on the HXRB ACF entail that the local volume emissivity of low luminosity sources clustered like normal galaxies

is bound to be $\rho_{x}(2-10 \mathrm{keV}) \lesssim 6 \times 10^{38} \mathrm{erg} \mathrm{s}^{-1} \mathrm{Mpc}^{-3}$. This value is half of that derived from the cross-correlation of the background with nearby galaxies and even lower than the estimate obtained through the XRB dipole (Jahoda et al. 1992). However the errors associated to Jahoda et al. (1992) estimates of local volume emissivity are so large that they are consistent at $1 \sigma$ with our limit. Moreover as pointed out by Jahoda (private communication) their estimate could be affected both by uncertainties in modelling the relationship between fluctuations in galaxy counts and XRB intensity as well as by the overdensity of the local universe.

\section{DISCUSSION AND CONCLUSIONS}

The smoothness of the SXRB significantly constrains the clustering scale, the clustering evolution and the contribution to the background of soft spectrum AGNs. The limits discussed in Section 3.1 can be straightforwardly compared with the results derived from optical surveys of QSOs (Iovino, Shaver \& Cristiani 1991; Boyle 1991; 
Andreani \& Cristiani 1992), because there is evidence that soft X-ray and optical selections tend to single out the same class of AGNs (Shanks et al. 1991; Setti 1991).

Recent analyses of large samples of optically selected QSOs have produced consistent values of the clustering scale $12 \lesssim r_{0} \lesssim 20$ Mpc (Iovino, Shaver \& Cristiani 1991; Boyle 1991; Andreani \& Cristiani 1992). The same authors also agree on the fact that there is evidence of evolution of the correlation function with $\epsilon \geq-1.2$, a constant comoving clustering scale being slightly favoured.

The limits derived in Section 3.1 are compatible with the optical results. It is interesting to notice that clustering with $r_{0}=20 \mathrm{Mpc}$ and $\epsilon=-1.2$ would imply that soft spectrum AGNs produce less than $40 \%$ of the SXRB and that the limit shifts to $50 \%$ if $r_{0}=12 \mathrm{Mpc}$. Indeed plausible models that are consistent with a number of observations, such as the local luminosity function, ROSAT and EINSTEIN Observatory source counts, redshift distributions and spectral properties of soft X-ray selected AGNs, predict an AGN contribution to the SXRB in the range between 30 to $50 \%$ (Franceschini et al. 1992). Thus, in the case of AGNs, clustering evolution with $\epsilon=-1.2$ is just on the verge of producing a too large ACF. Whatever the origin of the remaining background is, its sources must cluster rather weakly. Galaxy clusters and ASF galaxies are obvious candidates to produce the remaining $\sim 50 \%$ of the SXRB. Galaxy clusters are known to cluster on large scale, the values of $r_{0}$ found by various authors ranging from $r_{0} \approx 50$ to $r_{0} \approx 30 \mathrm{Mpc}$ (Bahcall \& Soneira 1983; Postman, Geller \& Huchra 1986; Sutherland 1988; Sutherland \& Efstathiou 1991; Bahcall \& West 1992). As a consequence their contribution to the background is bound to be small $(f \lesssim 15 \%)$, unless they are very extended and of low surface brightness. On the other hand ASF galaxies could yield a significant fraction of the SXRB, because they probably cluster on a scale comparable (possibly smaller, cfr. Efstathiou et al. 1991) to that of normal galaxies.

It is worth noticing that the constraints on both AGNs and ASF galaxies have been obtained under the hypothesis that any other contribution to the background 
is smoothly distributed in the sky. Therefore constraints on clustering are expected to be even more stringent than those derived above. For instance let us consider the case in which AGNs and ASF galaxies produce together a fraction $\mathrm{f} \simeq 80 \%$ of the SXRB (leaving room for contributions of galaxy clusters and galactic stars). Let us also assume that AGNs cluster with $r_{0}=12 \mathrm{Mpc}$ and $\epsilon=-1.2$ and give about $50 \%$ of the background; as a consequence they would saturate the autocorrelation level allowed by ROSAT limits (cfr. Figure 3). Then ASF galaxies giving the residual fraction $f \simeq 30 \%$ are bound to have $r_{0} \lesssim 10 \mathrm{Mpc}$ even in the case $\epsilon=0$ [cf. Figure 5 and eq. (9)].

As for the HXRB, Mushotzky \& Jahoda (1992) claimed a possible detection of positive autocorrelations on large scales, $\theta \geq 6^{\circ}$. This signal could well be due to nearby, hard spectrum AGNs clustered with $r_{0} \approx 20 \mathrm{Mpc}$. Moreover, if the clustering evolution is confined to $\epsilon \geq-1.2$, AGNs could also produce $\sim 60 \%$ of the HXRB, without violating the presently available limits on autocorrelations on scales of a few degrees (Martín-Mirones et al. 1991; Carrera et al. 1991; 1992). Of course these AGNs are bound to give a negligible contribution to the SXRB; this could be the result of an absorption increasing with increasing redshift (Franceschini et al. 1992).

As pointed out by Danese, De Zotti \& Andreani (1992) and by Carrera \& Barcons (1992), such a level of clustering cannot significantly affect the probability density distribution of the deflections from the mean, $P(D)$ observed by HEAO 1 A-2 (Shafer 1983) as well as by Ginga (Warwick \& Stewart 1989).

Clustering of galaxy clusters yields $W\left(6^{\circ}\right) \lesssim 1 \times 10^{-5}$, if $r_{0} \approx 30-50 \mathrm{Mpc}$, as found in optical surveys (Bahcall \& West 1992).

An alternative explanation of the possible detection of large scale autocorrelations requires that the local volume emissivity of the low luminosity sources is $\rho_{x}(2-$ $10 \mathrm{keV}) \approx 6 \times 10^{38} \mathrm{erg} \mathrm{s}^{-1} \mathrm{Mpc}^{-3}$.

Conversely the above value can be also interpreted as an upper limit to the local volume emissivity leading to the conclusion that the sources contributing the 
bulk of the HXRB are not local. This is somewhat in contrast with the findings by Jahoda et al. (1992) (see above §4) and Lahav et al. (1992), who claim a much larger local volume emissivity. Apart from the large uncertainties associated to their estimates, the only possible way to make their and our results consistent is that the low luminosity sources responsible for the local emissivity at the level claimed by these authors are clustered on a typical scale $r_{\circ} \approx 6 \mathrm{Mpc}$, half of the value found for normal galaxies.

All in all, the results on ACF found by Mushotzky \& Jahoda (1992) significantly constrains the local volume emissivity.

Important developments in ACF studies should come out from the analysis of the ASTRO-D data. Imaging capabilities with resolutions of few arcminutes will be obtained by this experiment in the $2-10 \mathrm{keV}$ band and sources brighter than some $10^{-14} \mathrm{erg} \mathrm{cm}^{-2} \mathrm{~s}^{-1}$ will be detected (Inoue 1992). We have computed the expected ACF in the hypothesis that the bulk of the HXRB is produced at substantial redshifts (the cumulative fraction of the effective volume emissivity has been reported in panel a) of Figure 1) and for a limiting flux of $S_{l} \sim 1 \times 10^{-13}$. Assuming $\gamma=1.8$ and following equation (9), we have found

$$
W(\theta) \approx g(\epsilon)\left(\frac{\theta}{5^{\prime}}\right)^{-0.8}\left(\frac{r_{0}}{12 M p c}\right)^{1.8}\left(\frac{f}{0.5}\right)^{2}
$$

where $\mathrm{g}(\epsilon)$ is a function of the clustering evolution; $\mathrm{g}(\epsilon)$ is $4 \times 10^{-3}, 1 \times 10^{-2}$ and $8 \times 10^{-2}$ for $\epsilon=0, \epsilon=-1.2$ and $\epsilon=-3$ respectively. The availability of ACF data on large and small angular scales would then allow to extract important information on the clustering of sources contributing to the HXRB both in the local as well as in the high redshift universe.

Finally, it is worth noticing that the limits on clustering derived from SXRB and HXRB correlations suggest that soft, highly luminous AGNs and hard, less luminous AGNs have rather similar clustering properties, with scales not much larger than that 
of the galaxies, and evolution at least as steep as implied by the comoving clustering model, i.e. $\epsilon \geq-1.2$.

Acknowledgements We thank X. Barcons, F. Carrera, G. Hasinger and G. Setti for enlightening discussions. Comments and suggestions of an anonimous referee and of K. Jahoda have been extremely helpful. Work supported in part by MURST, GNA/CNR and ASI. 


\section{REFERENCES}

Anderson, S.F., Windhorst, R.A., Maccacaro, T., Burstein, D., Franklin, B.E., Griffiths, R.E., Koo, D.C., Mathis, D.F., Morgan, W.A. \& Neuschafer, L.W. 1992, in X-ray Emission from Active Galactic Nuclei and the Cosmic X-ray Background, eds. W. Brinkmann \& J. Trümper, MPE Report 235, p. 227

Andreani, P., \& Cristiani, S. 1992, ApJ, 398, L13

Bahcall, N.A., \& Chokshi, A. 1991, ApJ, 380, L9

Bahcall, N.A., \& Soneira, R.M. 1983, ApJ, 270, 20

Bahcall, N.A., \& West, M.J. 1992, ApJ, 392, 419

Barcons, X., \& Fabian, A.C. 1989, MNRAS, 237, 119

Blanchard, A., Wachter, K., Evrard, A.E., \& Silk, J. 1992, ApJ, 391, 1

Boldt, E.A. 1987, Phys. Rep., 146, 215

Boldt, E. 1992, in The X-ray Background, eds. X. Barcons \& A.C. Fabian (Cambridge University Press), 115

Boller, Th., Meurs, E.J.A., Brinkmann, W., Fink, H., Zimmermann, U., \& Adorf, H.M. 1992, in X-ray Emission from Active Galactic Nuclei and the Cosmic X-ray Background, eds. W. Brinkmann \& J. Trümper, MPE Report 235, p.231

Bookbinder, J., Cowie, L.L., Krolik, J.H., Ostriker, J.P., \& Rees, M. 1980, ApJ, 237, 647

Boyle, B.J. 1991, in Texas-ESO/CERN Conference on Relativistic Astrophysics, in press

Carrera, F.J., \& Barcons, X. 1992, MNRAS, 257, 507

Carrera, F.J., Barcons, X., Butcher, J.A., Fabian, A.C., Stewart, G.C., Warwick, R.S., Hayashida, K. \& Kii, T. 1991, MNRAS, 249, 698

Carrera, F.J., Barcons, X., Butcher, J.A., Fabian, A.C., Stewart, G.C., Toffolatti, L., Warwick, R.S., Hayashida, K., Inoue, H., \& Kondo, H. 1992, MNRAS, in press Cavaliere, A., Menci, N., \& Burg, R. 1992, preprint 
Cen, R., \& Ostriker, J. 1992, ApJ, 393, 22

Danese, L., De Zotti, G., \& Andreani, P. 1992, in The X-ray Background, eds. X. Barcons \& A.C. Fabian (Cambridge University Press), 61

Danese, L., De Zotti, G., Franceschini, A., \& Toffolatti, L. 1987, ApJ, 318, L15

De Zotti, G., Persic, M., Franceschini, A., Danese, L., Palumbo, G.G.C., Boldt, E.A., \& Marshall, F.E. 1990, ApJ, 351, 22

Efstathiou, G., Burnstein, G., Katz, N., Tyson, J.A., \& Guhathakurta, P. 1991, ApJ, $380, \mathrm{~L} 47$

Efstathiou, G., \& Rees, M.J. 1988, MNRAS, 230, 5P

Fabbiano, G., Kim, D.W., \& Trinchieri, G. 1992, ApJS, in press

Fabian, A.C. 1972, Nature Phys. Sci., 237, 19

Franceschini, A., Danese, L., De Zotti, G., \& Toffolatti, L. 1988, MNRAS, 233, 157

Franceschini, A., Martín-Mirones, J.M., Danese, L., \& De Zotti, G. 1992, MNRAS, in press

Georgantopoulos, I., Stewart, G.C., Griffiths, R.E., Shanks, T., \& Boyle, B. 1992, in $\mathrm{X}$-ray Emission from Active Galactic Nuclei and the Cosmic X-ray Background, eds. W. Brinkmann \& J. Trümper, MPE Report 235, p. 368

Gioia, I.M., Henry, J.P., Maccacaro, T., Morris, S.L., Stocke, J.T., \& Wolter, A. 1990, ApJ, 356, L35

Griffiths, R.E., \& Padovani, P. 1990, ApJ, 360, 483

Hacking, P., Condon, J.J., \& Houck, J.R. 1987, ApJ, 316, L15

Hasinger, G. 1992, in The X-ray Background, eds. X. Barcons \& A.C. Fabian (Cambridge University Press), 229

Hasinger, G., Schmidt, M., \& Trümper, J. 1991, A\&A, 246, L2

Inoue, H., 1992, in The X-ray Background, eds. X. Barcons \& A.C. Fabian (Cambridge University Press), 286

Iovino, A., Shaver, P.A., \& Cristiani, S. 1991, in The Space Distribution of Quasars, ed. D. Crampton, ASP Conference Series n. 21, p. 202 
Jahoda, K., Lahav, O., Mushotzky, R.F., Boldt, E.A. 1991, ApJ, 378, L37

Jahoda, K., Lahav, O., Mushotzky, R.F., Boldt, E.A. 1992, ApJ, 399, L107

Kaiser, N. 1986, MNRAS, 222, 323

Lahav, O. 1992, in The X-ray Background, eds. X. Barcons \& A.C. Fabian (Cambridge University Press), 102

Lahav, O., Fabian, A.C., Barcons, X., Boldt, E., Butcher, J., Carrera, F.J., Jahoda, K., Miyaji, T., Stewart, G.C., \& Warwick, R.S. 1992, Nature, in press

Maccacaro, T., Della Ceca, R., Gioia, I.M., Morris, S.L., Stocke J.T., \& Wolter, A. 1991, ApJ, 374, 117

Maddox, S.J., Efstathiou, G., Sutherland, W.J., \& Loveday, J. 1990, MNRAS, 242, $43 \mathrm{p}$

Marshall, F.E., Boldt, E.A., Holt, S.S., Miller, R.B., Mushotzky, R.F., Rose, L.A., Rothschild, R.E., \& Serlemitsos, P.J. 1980, ApJ, 235, 4

Martínez-González, E., \& Sanz, J.L. 1991, MNRAS, 248, 816

Martín-Mirones, J.M., De Zotti, G., Boldt, E.A., Marshall, F.E., Danese, L., Franceschini, A., \& Persic, M. 1991, ApJ, 379, 507

Mather, J.C. et al. 1990, ApJ, 354, L37

McCammon, D., \& Sanders, W.T. 1990, ARA\&A, 28, 657

Micela, G., Harnden, F.R., J.R., Rosner, R., Sciortino, S., \& Vaiana, G.S. 1991, ApJ, 380,495

Mushotzky, R., \& Jahoda, K. 1992, in The X-ray Background, eds. X. Barcons \& A.C. Fabian (Cambridge University Press), 80

Peebles, P.J.E. 1980, The Large-Scale Structure of the Universe (Princeton: Princeton University Press)

Persic, M., De Zotti, G., Boldt, E.A., Marshall, F.E., Danese, L., Franceschini, A., \& Palumbo, G.G.C. 1989, ApJ, 336, L47

Postman, M., Geller, M.J., \& Huchra, J.P. 1986, AJ, 91, 1267 
Primini, F.A., Murray, S.S., Huchra, J., Schild, R., Burg, R., \& Giacconi, R. 1991, ApJ, 374, 440

Rephaeli, Y., Gruber, D., Persic, M., \& MacDonald, D. 1991, ApJ, 380, L59

Rees, M.J. 1986, in The Structure and Evolution of Active Galactic Nuclei, eds. G. Giuricin, F. Mardirossian, M. Mezzetti, \& M. Ramella (Dordrecht: Reidel), p. 447

Schwartz, D.A., Boldt, E.A., Holt, S.S., Serlemitsos, P.J., \& Bleach, R.D. 1971, Nature Phys. Sci., 233, 110

Sebok, W.L. 1986, ApJS, 62, 301

Setti, G. 1991, in Frontiers of X-ray Astronomy, Proceedings of the 28th Yamada Conference, eds. Y. Tanaka \& K. Koyama (Universal Academy Press), 663

Shafer, R.A. 1983, Ph. D. thesis, University of Maryland

Shanks, T., Georgantopoulos, I., Stewart, G.C., Pounds, K.A., Boyle, B.J., \& Griffiths, R.E. 1991, Nature, 353, 315

Soltan, A.M. 1991, MNRAS, 250, 241

Stewart, G.C., Fabian, A.C., Terlevich, R.J., \& Hazard, C. 1982, MNRAS, 200, 61p

Sutherland, W.J. 1988, in IAU Symposium 130, Large Scale Structures of the Universe, ed J. Audouze, M.C. Pelletan, and A. Szalay (Dordrect: Kluwer), p. 538

Sutherland, W.J., \& Efstathiou, G. 1991, MNRAS, 248, 159

Turner, E.L., \& Geller, M.J. 1980, ApJ, 236, 1

Tyson, J.A. 1988, AJ, 96, 1

Wolfe, A.M., \& Burbidge, G.R. 1970, Nature, 228, 1170

Wu, X., Hamilton, T., Helfand, D.J., \& Wang, Q. 1991, ApJ, 379, 564 


\section{FIGURE CAPTIONS}

Figure 1 Predicted cumulative fraction of the ACF $(W(\theta)$, continuous line $)$ and of the effective volume emissivity ( $j_{\text {eff }}$, short-dashed line) per unit logarithmic redshift interval for hard X-ray selected AGNs. The relevant parameters and the angular scale are indicated in each panel.

Figure 2 Same as in Figure 1 but for soft X-ray selected sources. Panels a) and b) refer to AGNs while panels c) and d) refer to Active Star Forming galaxies (see Section 3.2).

Figure 3 Constraints on the fraction $(f)$ of the total background contributed by unresolved AGNs as a function of the clustering scale, $r_{0}$. The plotted curves, following the scaling law of equation (9), are obtained by saturating the $3 \sigma$ upper limit on $W\left(9^{\prime}\right)$ from ROSAT deep exposures (Hasinger 1992). The different curves refer to different values of the clustering evolution parameter $\epsilon$ as indicated by the labels. The current unresolved fraction of the SXRB is indicated by the dotted-longdashed line $(f=0.6)$ (see text for details).

Figure 4 Same as in Figure 3 but using the $3^{\prime}$ upper limit obtained by Soltan (1991) from the analysis of Einstein IPC data. The dotted-long-dashed line again indicate the fraction of the unresolved background $(f=0.8)$ at the limit of the Einstein Deep Survey.

Figure 5 Same as in Figure 3 but for ASF galaxies. 